\title{
Optimal Transport Flows for Distributed Production Networks
}

\author{
Julius B. Kirkegaard and Kim Sneppen \\ Niels Bohr Institute, University of Copenhagen, 2100 Copenhagen, Denmark
}

(Received 8 October 2019; accepted 23 April 2020; published 22 May 2020)

\begin{abstract}
Network flows often exhibit a hierarchical treelike structure that can be attributed to the minimization of dissipation. The common feature of such systems is a single source and multiple sinks (or vice versa). In contrast, here we study networks with only a single source and sink. These systems can arise from secondary purposes of the networks, such as blood sugar regulation through insulin production. Minimization of dissipation in these systems leads to vascular shunting, a single vessel connecting the inlet and outlet. We show instead how optimizing the transport time yields network topologies that match those observed in the insulin-producing pancreatic islets. These are patterns of periphery-to-center and center-to-periphery flows. The obtained flow networks are broadly independent of how the flow velocity depends on the flow flux, but continuous and discontinuous phase transitions appear at extreme flux dependencies. Lastly, we show how constraints on flows can lead to buckling of the branches of the network, a feature that is also observed in pancreatic islets.
\end{abstract}

DOI: 10.1103/PhysRevLett.124.208101

Transport networks are essential for life to function on large multicellular scales. In vertebrates, blood flow delivers energy and nutrients and removes waste through the branched network of the vascular system. The separation of vessels into arteries and veins ensures that oxygen-rich and oxygen-depleted parts of the network are kept separate. In plants, the separation into xylem and phloem provides a similar function. In nature, these systems typically exhibit treelike hierarchical structures, as, e.g., in the aorta, which splits into increasingly smaller arteries all the way to capillaries, the smallest vessels of the circulatory system. By considering the arterial system and venial system independently, this tree structure can be understood as the minimization of dissipation of the blood flow through the system [1-3]. Furthermore, loop-redundant treelike structures, as is evident from, e.g., the veins of a tree leaf, can be understood as robustness against damage or fluctuating needs $[4,5]$. Treelike structures are observed not just in vascular networks, but also, for instance, in both natural and artificial river networks $[4,6]$.

In modeling the arterial system separate from the venial system, the heart is considered the source of the blood flow and the body cells sinks, whereas the roles are reversed in venous systems. Of course, the vascular system is in reality a single connected flow system, but it is this division into oxygen-rich and oxygen-depleted blood

Published by the American Physical Society under the terms of the Creative Commons Attribution 4.0 International license. Further distribution of this work must maintain attribution to the author(s) and the published article's title, journal citation, and DOI. which allows the dissipation minimization to yield tree structures [Fig. 2(a)]. Minimizing dissipation in systems with a single source and sink (or a few of each) leads to vascular shunting [7]: a singular vessel leading from the source to sink [Fig. 2(b)].

In this Letter, we propose an alternative minimization problem on flow networks with a single source and sink that avoids shunting and leads flow to all cells. In particular, we consider the minimization of flow time between the inlet or outlet and all cells of the systems. Such time minimization will be relevant when fast synchronization or transfer of information between the nodes is important. Likewise, time from the inlet to cells can be seen as a local proxy for the concentration of products that enter the system (e.g., oxygen in blood) and decay as a function of time. In a similar fashion, time from cells to the outlet could be seen as a proxy for efficient removal of metabolites produced by cells in the system.

As a specific biological exemplar, we consider the islets of Langerhans in the pancreas. In these islets, beta cells release insulin and alpha cells glucagon into the blood stream based on blood glucose levels [8,9]. Here there is no need for an arterial-venous separation, as oxygen plays only a secondary role, the production and delivery of hormones being of primary concern. The average blood flow velocity in Langerhans islets is $\sim 1.4 \mathrm{~mm} / \mathrm{s}$ [10], implying that transport across an islet takes about $\sim 0.5 \mathrm{~s}$. Activity of both alpha and beta cells is pulsatile, and in vitro experiments show coherent oscillations of whole islets with periods down to $3 \mathrm{~s} \mathrm{[11].} \mathrm{In} \mathrm{order} \mathrm{for} \mathrm{the} \mathrm{organism} \mathrm{to}$ utilize coherent release of insulin downstream of islets, it is therefore plausible that time optimization on the subsecond scale is functional. 


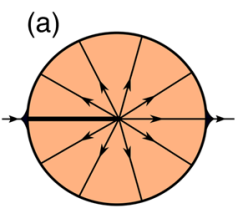

(b)

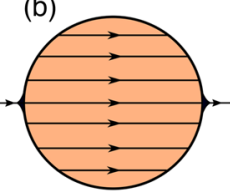

(c)

(d)

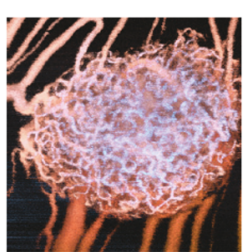

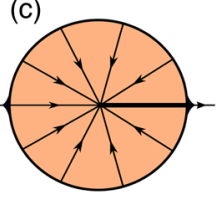

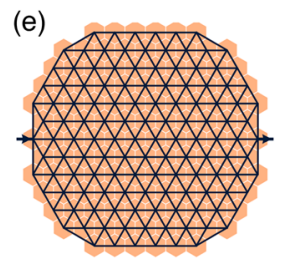

FIG. 1. Vasculature of pancreatic islets. Idealized center-toperiphery flow (a), left-right symmetric flow (b), and peripheryto-center flow (c). (d) Microscopy of vasculature, from Ref. [10], showing the high degree of tortuousness in the vasculature of pancreatic islets. (e) The network structure considered. This is constructed by taking a circular subsection of a hexagonal grid of nodes. Neighbors are induced from a Delaunay triangulation. Each edge has a conductivity $C_{e}$ associated to it, which are the parameters to be optimized over. The inlet and outlet are shown by arrows.

The vasculature of pancreatic islets differs from species to species. In particular, various topologies have been observed: periphery-to-center flow, straight through, and center-to-periphery flow, as idealized in Figs. 1(a)-1(c) [12-14]. For instance, in rodents, the center-to-periphery topology is the most common [13]. Furthermore, the vasculature of these islets is often very tortuous compared to the vasculature of other organs $[10,15]$ as shown in Fig. 1(d).

Model.-To study systems of blood flow optimization, we consider, as in previous studies [1-5], flows on networks. While pancreatic islets indeed can have more than one inlet and outlet, we simplify the system and consider the network shown in Fig. 1(e). Regardless, our approach works for any number of sources and sinks. In Fig. 1(e), cells are represented by hexagons, and edges between these indicate where fluid may flow. The inlet and outlet are indicated by arrows.

This specific graph has $n_{N}=130$ nodes and $n_{E}=357$ edges, each edge $e$ of the graph having a length $L_{e}$ and a conductivity $C_{e}$. An $n_{E} \times n_{N}$ oriented incident matrix $\Delta$ of the graph gives each edge a unique direction, and we can thus tie to each edge a (signed) flow $F_{e}$. We furthermore define the source vector $S$ with $S_{\text {source }}=1, S_{\text {sink }}=-1$, and $S_{i}=0$ elsewhere and require that the flow obeys Kirchoff's current law

$$
\Delta^{T} \boldsymbol{F}=\boldsymbol{S} .
$$

Since $n_{N}<n_{E}$, the flow is far from determined by this condition alone.

To make the flow well defined, we require that it is derivable from a potential based on the effective conductivities:

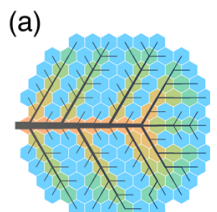

(b)

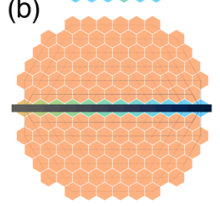

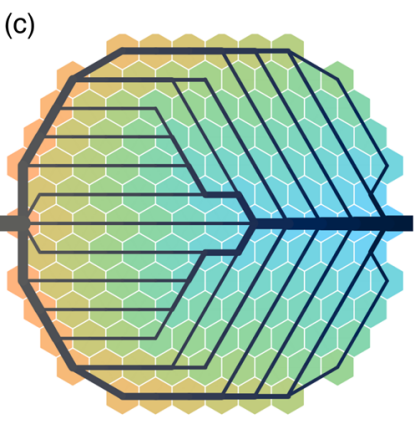

FIG. 2. Optimized network structures. (a) Minimal dissipation network with sinks at all nodes. (b) Minimal dissipation network with a single sink at the edge. (c) Per-node time-minimizing network with a single sink at the edge. Cell colors indicate the average time to the outlet(s). Flow lines are colored by pressure, their thickness indicating the (square root of) flow magnitude.

$$
\boldsymbol{F}=\boldsymbol{C}^{\mathrm{eff}} \Delta \boldsymbol{p},
$$

where $p_{i}$ is the potential (pressure) defined at the nodes and $C^{\text {eff }}$ is a diagonal matrix with entries $C_{e e}^{\text {eff }}=$ $C_{e} / L_{e}$. Combining Eqs. (1) and (2), we can solve for the potential as

$$
\boldsymbol{p}=\left[\Delta^{T} \boldsymbol{C}^{\mathrm{eff}} \Delta\right]^{\dagger} \boldsymbol{S},
$$

where $\dagger$ denotes the pseudoinverse. While the system of equations is singular, it can be solved, as stated, by the pseudoinverse if $\sum_{i} S_{i}=0$, which is indeed the case here. From the potentials $\boldsymbol{p}$, the flows are immediately obtained from Eq. (2).

The total power dissipation of the system is $P=$ $\sum_{e} F_{e}^{2} / C_{e e}^{\mathrm{eff}}$ [1-3]. As mentioned, minimizing this term leads to tree topologies for a single source and sinks everywhere $\left[S_{\text {source }}=1, S_{i}=-1 /\left(n_{N}-1\right)\right.$ elsewhere $]$. This optimum on our network topology is shown in Fig. 2(a). The minimization is done under constant "material cost" $\sum_{e} L_{e} C_{e}^{\gamma}$, and the treelike structures are obtained for $\gamma<1$. In the optimum, the conductivities scale with the flow as [16]

$$
C_{e} \sim\left|F_{e}\right|^{2 /(1+\gamma)}
$$

i.e., a larger conductivity is needed where there is a lot of flow.

Figure 2(b) shows the "vascular shunting" solution obtained by minimizing power dissipation in the network with just a single source and single sink. This is also, naturally, the time-minimizing network for flow between the source and the sink.

We are interested in network structures that visit all nodes in an "optimal" way. Indeed, proper distribution of vessels is far more important in pancreatic islets and other systems than the (potentially minuscule) power being dissipated. As such, we consider instead graphs that 
minimize the average time for the product (e.g., insulin) being produced at the nodes to reach the outlet (the opposite inlet-centric definition will be discussed later). This time-optimized graph we define as follows: Denote for each node $T_{i}$ the average time taken from that node for the product to reach the outlet. The average time is thus

$$
\langle T\rangle=\frac{1}{n_{N}} \sum T_{i}
$$

where $T_{i}$ is found by the recursive relation which follows by letting the product flow in proportion to the fluid flow:

$$
T_{i}=\frac{\sum_{j \in \mathcal{O}_{i}}\left|F_{i j}\right|\left(T_{j}+T_{i j}\right)}{\sum_{j \in \mathcal{O}_{i}}\left|F_{i j}\right|},
$$

with the special case $T_{\text {sink }}=0$. This is a linear equation for $T_{i}$, where $\mathcal{O}_{i}$ is the set of nodes that are outgoing from node $i$. Whether an edge $e$ is outgoing from node $i$ can be identified by the criteria $F_{e} \Delta_{e i}>0 . T_{i j}$, the time taken for the product to flow from node $i$ to neighboring node $j$, defines the physics of the system. We will fix this by the relation

$$
T_{i j} \sim \frac{L_{i j}}{\left|F_{i j}\right|^{\delta}} .
$$

Equation (7) states that the time spent for blood to flow over an edge of length $L_{i j}$ scales as a power law with the flux over that edge. This is the physical flow time spent under restrictions of a "building rule" requiring the conductivity to scale with the flux. To see this, consider that each blood vessel corresponds to a tube of a given radius $r_{e}$. We can then relate $F_{e} \sim v_{e} r_{e}^{2}$, where $v_{e}$ is the fluid velocity along the tube. Furthermore, in Poiseuille flow $C_{e} \sim r_{e}^{4}$, and thus $T_{i j}=T_{e}=L_{e} / v_{e} \sim L_{e} \sqrt{C_{e}} / F_{e}$. Equation (7) is then obtained by requiring $C_{e}$ to scale as a power law of $F_{e}$. Optimizing directly without this requirement yields solutions of vanishingly small conductivities everywhere, as in small tubes the liquid will have to move faster for the same flux $F$. Such solutions are severely dissipation inefficient, and our rule ensures a balance of global time optimization with local power efficiency.

In one interpretation, our rule can be seen as local power optimization under global material cost constraints: Eq. (4) states that, in power-optimal solutions of systems with a fixed material cost, the conductivities scale with the flux. If we assume that the material cost is kept fixed on a large scale on which power is exclusively optimized for, say, the entire vasculature of an organism, then locally (say, in organs), Eq. (4) specifies that, in order to be power efficient, a flux of size $F$ must be associated with a conductivity of size $|F|^{2 /(1+\gamma)}$. Equation (7) is then obtained by setting $\delta=\gamma /(1+\gamma)$. We discuss the situation in which material cost is kept constant on the local scale in
Supplemental Material [17]. Other approaches such as Pareto efficiency, could also be considered for jointly balancing power and time minimization.

Equations (5)-(7) define the optimization problem, which we solve by a momentum-based version of gradient descent and simulated annealing [17]. We note that the system has many local optima (akin to the optimization of dissipation) and that simulated annealing is not guaranteed to yield the true global optimum.

Periphery-center optima.-We begin by fixing $\delta=1 / 2$ and discuss variations later. The result of our minimization scheme is shown in Fig. 2(c). The single-source-sink system prevents self-similar branching solutions that are known from single-source, multiple-sink systems. Instead, the solution has only a few main branches, in particular, one at the periphery and one at the center. Interestingly, this pattern is one of the three idealized topologies [Figs. 1(a)-1(c)] of pancreatic islet blood flow observed in nature $[13,14]$.

In some species, such as rodents, the glucagon-producing alpha cells and the insulin-producing beta cells comprising the islets are heterogeneously distributed with the beta cells in the center and alpha cells at the periphery. It has thus been suggested that the order of the flow suggests intercellular communication and regulation [13], i.e., beta cells regulating alpha cells or vice versa, depending on the flow topology. Our results show a separate, but nonexclusive explanation, namely, that the patterns can appear due to an optimization of the flow itself, independent of any heterogeneous distribution of cells.

The equal distribution of flow to each node and the sparsity of the network in the optimum [Fig. 2(c)] is possible due to the choice of $\delta=1 / 2$ in Eq. (7). In fact, our model works for a broad range of $\delta$. To illustrate this, consider the simple network of three nodes shown in Fig. 3(a). Taking $1=F_{1}+F_{2}$, it follows that $2\langle T\rangle=$ $\left(1-F_{1}\right)^{1-\delta}+2 F_{1}^{1-\delta}+F_{1}^{-\delta}$, which is shown in Fig. 3(b) for various values of $\delta$. Minimizing $\langle T\rangle$, the optimal $F_{1}$ is shown in Fig. 3(c), which demonstrates that this system has a discontinuous phase transition at $\delta \approx 0.275$ and a continuous phase transition at $\delta=1$ in $F_{1}$. Between these
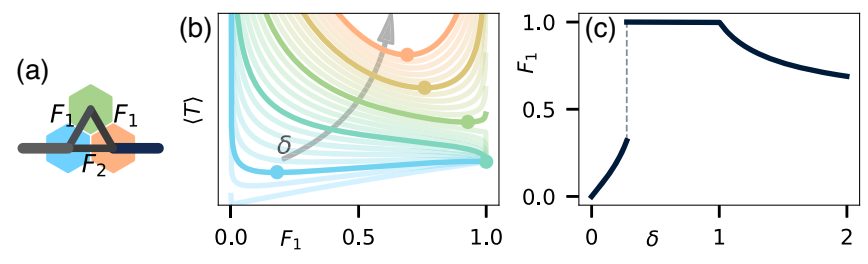

FIG. 3. Phase transition in triangle geometry. (a) Triangle geometry. We consider $S_{\text {source }}=1=F_{1}+F_{2}$. (b) Energy $(\langle T\rangle)$ landscape for various $\delta$. Circles indicate select minima. (c) Value of $F_{1}$ obtained by minimizing $\langle T\rangle$ for various scalings $\delta$. There is a discontinuous transition at $\delta \approx 0.275$ and a continuous transition at $\delta=1$. 

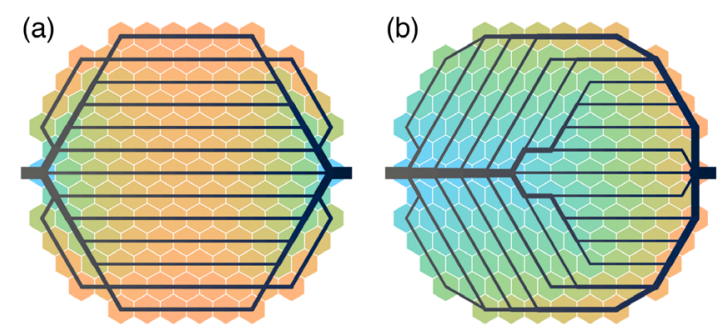

FIG. 4. Varying the importance $\alpha$ between time from nodes to the outlet and the inlet to nodes. (a) The left-right symmetric solution obtained at $\alpha=0.5$. (b) The solution in Fig. 2(c) is reversed for $\alpha=1.0$. Background color indicates $T^{1-\alpha} T_{r}^{\alpha}$.

values, the optimal $F_{1}$ is independent of $\delta$, which is the regime we study.

The discontinuous phase transition occurs because the left and top nodes in Fig. 3(a) have conflicting optima: The left node minimizes its time by having $F_{2}$ large, whereas the top node needs $F_{1}$ large. Large $\delta$ favor large $F_{1}$, because then a larger flow velocity compensates for the larger length of the upper branch. In contrast, a small $\delta$ favors the shorter path of the lower branch, while leaving only a small flux through the upper branch required to transport the product from the top node.

For more complex graphs such as the one we consider [Fig. 1(e)], the phase transition behavior is more complex but remains qualitatively similar, albeit with the lower transition pushed to even lower values of $\delta$ (see Supplemental Material [17] for examples). Our choice of $\delta=1 / 2$ lies safely within the regime, where the results are independent of the precise value of $\delta$ and where the resulting networks are sparse graphs. Thus, our results are to a large degree independent of the scaling in Eq. (7).

The left-right asymmetry and thus the periphery-tocenter flow in Fig. 2(c) stems from the fact that we are minimizing the time for the product to reach the outlet from the nodes. The large collection branch emerging from the center thus minimizes the time for many nodes by providing a fast route. Had we instead minimized the time from the source to the nodes $\left\langle T_{r}\right\rangle$, the solution would be reversed, since it would be important to reach the nodes fast. Indeed, this opposite situation, with flow from center to periphery, is the most commonly observed topology in rodents [13] and could perhaps hint that the time for "information" (e.g., glucose levels) to reach the cells is more important than the product to exit the islet.

Naturally, the combination of time from the inlet to nodes and from nodes to the outlet can also be considered. For instance, the weighted geometric mean of the two $\left\langle T_{c}\right\rangle=\left\langle T^{1-\alpha} T_{r}^{\alpha}\right\rangle(0 \leq \alpha \leq 1)$ can also be optimized over. The arithmetic mean also works but is less robust against variations at the edges of the systems. Figure 4(a) demonstrates that at $\alpha=0.5$ the solution becomes left-right symmetric, yielding an islet topology similar to that in Fig. 1(b). Taking $\alpha=1$ [Fig. 4(b)] left-right mirrors the original solution, showing that the three distinct topologies of pancreatic islets (Fig. 1) can be obtained simply by varying this parameter.

Minimal flow constraints. - Various constraints can additionally be added to the system. For instance, while the optimum in Fig. 2(c) does indeed distribute flow to all nodes, some nodes see much more fluid flowing through them than others. To equalize this, one can add flow constraints to the optimization problem. The flow through a node $i$ with $S_{i}=0$ is

$$
\mathcal{F}=\sum_{j \in \mathcal{O}_{i}}\left|F_{i j}\right|,
$$

and we can then require $\mathcal{F} \geq \mathcal{F}_{m}$ for all nodes for a given value of $\mathcal{F}_{m}$. With increasing $\mathcal{F}_{m}$, the flow will have to adapt in order to provide the required amount of blood to each cell, until $\mathcal{F}_{m}$ becomes so large that this is no longer possible.

This situation could model growth of the pancreatic islets. During growth, if the blood flow source cannot keep up, each cell or node will see a decrease in flow permeating them. In this way, growth in networks flows can be emulated by a decreasing $S_{\text {source }}$ [3]. At the same time, a minimum amount of flow might be required at each cell.
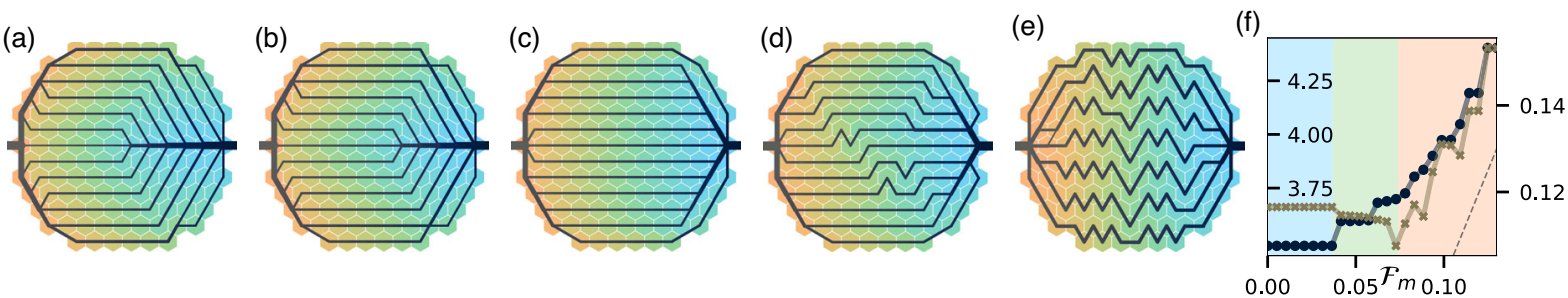

FIG. 5. Optimal graphs under minimal flow constraints. (a) At $\mathcal{F}_{m}=0.042$, the network in Fig. 2(c) loses its front collection channels. (b) The collection point starts moving to the right as $\mathcal{F}_{m}$ is increased to 0.062 . (c) At $\mathcal{F}_{m}=0.073$, the collection point has moved to the far right. (d) One horizontal vessel has been removed by introducing kinks at $\mathcal{F}=0.078$, which increases the flow in the remaining vessels. (e) Buckling increases; $\mathcal{F}_{m}=0.125$. As $\mathcal{F}_{m} \rightarrow 1$, the global optimum becomes a Hamiltonian path through the nodes. (f) In dark blue (left axis), the average time $\langle T\rangle$ is shown as a function of $\mathcal{F}_{m}$. The average flow through the nodes $\langle\mathcal{F}\rangle$ is shown in light brown (right axis). The dashed line shows $f(x)=x$, which matches the slope of $\langle\mathcal{F}\rangle$ in the buckling section. Background colors denote section: global optimum in blue, unbuckled solution in green, and buckled in red. 
While our time measure is a global construct, this is a local constraint on the fluxes, which could be relevant if, for instance, insulin production or transmission depends directly on the flux. Likewise, a large flux could ensure accurate estimation of glucose levels, under the assumption that these sensors metabolise only a fraction of the glucose available in the blood stream. Other constraints, such as the local concentration of glucose or insulin, could also be tested on the system.

Obtained minima are shown in Fig. 5 under variations of $\mathcal{F}_{m}$. The optimal configuration in Fig. 2(c) has $\min _{i} \mathcal{F}=0.041$, and, thus, for any $\mathcal{F}_{m}$ smaller than this, the same solution is obtained [blue section in Fig. 5(f)]. As $\mathcal{F}_{m}$ is increased above this, the network adapts to more equally divide the flow. First, the two middle branches are lost [Fig. 5(a)], and then the collection point starts moving toward the outlet [Fig. 5(b)]. In the end of this process [green section in Fig. 5(f)], the collection branch has moved all the way to the right [Fig. 5(c)]. As can be seen in Fig. 5(f), naturally, the average time $\langle T\rangle$ increases as $\mathcal{F}_{m}$ increases. Figure 5(f) also shows that $\langle\mathcal{F}\rangle_{i}$ decreases rapidly. So, as this reordering occurs, the minimum flow rate increases at the expense of the average flow rate.

As $\mathcal{F}_{m}$ is increased further than reordering can accommodate for, buckling occurs [Fig. 5(d)], the degree of which increases as $\mathcal{F}_{m}$ is increased [Fig. 5(e)]. As soon as buckling occurs, $\langle\mathcal{F}\rangle$ starts increasing as seen in Fig. 5(f). This increase scales linearly with $\mathcal{F}_{m}$; i.e., the average flow rate, after buckling, stays at a fixed level above $\mathcal{F}_{m}$. We note that the noise in $\langle\mathcal{F}\rangle$ in Fig. 5(f) most likely indicates that our optimization scheme in some cases fails to find the best optimum but ends in neighboring local minima.

As shown in Fig. 1(d), pancreatic islets do indeed have a severely tortuous vasculature [10] similar to that obtained in Fig. 5. The mechanical reason for buckling in real islets could be due to growth-induced buckling [15]. Our analysis indicates circumstances under which such buckling could actually be of benefit.

This project has received funding from the European Research Council (ERC) under the European Union's Horizon 2020 Research and Innovation Program, Grant Agreement No. 740704, and the Danish National Research Foundation, Grant No. DNRF116.

[1] S. Bohn and M. O. Magnasco, Structure, Scaling, and Phase Transition in the Optimal Transport Network, Phys. Rev. Lett. 98, 088702 (2007).

[2] D. Hu and D. Cai, Adaptation and Optimization of Biological Transport Networks, Phys. Rev. Lett. 111, 138701 (2013).
[3] H. Ronellenfitsch and E. Katifori, Global Optimization, Local Adaptation, and the Role of Growth in Distribution Networks, Phys. Rev. Lett. 117, 138301 (2016).

[4] E. Katifori, G. J. Szöllosi, and M. O. Magnasco, Damage and Fluctuations Induce Loops in Optimal Transport Networks, Phys. Rev. Lett. 104, 048704 (2010).

[5] F. Corson, Fluctuations and Redundancy in Optimal Transport Networks, Phys. Rev. Lett. 104, 048703 (2010).

[6] P. S. Dodds, Optimal Form of Branching Supply and Collection Networks, Phys. Rev. Lett. 104, 048702 (2010).

[7] T.-H. Nguyen, A. Eichmann, F. Le Noble, and V. Fleury, Dynamics of vascular branching morphogenesis: The effect of blood and tissue flow, Phys. Rev. E 73, 061907 (2006).

[8] P. M. Pour, J. Standop, and S. K. Batra, Are islet cells the gatekeepers of the pancreas? Pancreatology 2, 440 (2002).

[9] H. Hong, J. Jo, and S. J. Sin, Stable and flexible system for glucose homeostasis, Phys. Rev. E 88, 032711 (2013).

[10] C. Berclaz, D. Szlag, D. Nguyen, J. Extermann, A. Bouwens, P. J. Marchand, J. Nilsson, A. Schmidt-Christensen, D. Holmberg, A. Grapin-Botton, and T. Lasser, Label-free fast 3D coherent imaging reveals pancreatic islet microvascularization and dynamic blood flow, Biomed. Opt. Express 7, 4569 (2016).

[11] M. Valdeolmillos, R. M. Santos, D. Contreras, B. Soria, and L. M. Rosario, Glucose-induced oscillations of intracellular $\mathrm{Ca} 2+$ concentration resembling bursting electrical activity in single mouse islets of Langerhans, FEBS Lett. 259, 19 (1989).

[12] H. Shimokawa, The order of islet microvascular cellular perfusion is B-A-D in the perfused rat pancreas, J. Clin. Invest. 82, 350 (1988).

[13] L. R. Nyman, K. S. Wells, W. S. Head, M. McCaughey, E. Ford, M. Brissova, D. W. Piston, and A. C. Powers, Realtime, multidimensional in vivo imaging used to investigate blood flow in mouse pancreatic islets, J. Clin. Invest. 118, 3790 (2008).

[14] Y. El-gohary and G. Gittes, Structure of islets and vascular relationship to the exocrine pancreas, Pancreapedia (2017).

[15] J. B. Kirkegaard, B. F. Nielsen, A. Trusina, and K. Sneppen, Self-assembly, buckling \& density-invariant growth of three-dimensional vascular networks, J. R. Soc. Interface 16, 20190517 (2019).

[16] Briefly, minimising $\sum_{e} L_{e} F_{e}^{2} / C_{e}$ under constant $\sum_{e} L_{e} C_{e}^{\gamma}$ by the method of Lagrange multipliers leads to $-F_{e}^{2} / C_{e}^{2}+$ $\lambda \gamma C_{e}^{\gamma-1}=0$ from which the scaling follows. See e.g., Ref. [1] for details.

[17] See Supplemental Material http://link.aps.org/supplemental/ 10.1103/PhysRevLett.124.208101 for details of the numerical optimisation scheme, discussion of varying $\delta$ in the full system, and discussion of keeping material cost fixed within the system, which includes Ref. [18].

[18] G. H. Golub and V. Pereyra, The differentiation of pseudoinverses and nonlinear least squares problems whose variables seperate, SIAM J. Num. Anal. 10, 413 (1973). 